\title{
El juego como metodología de investigación e intervención con niñas, niños y adolescentes ${ }^{\star}$
}

\author{
Verónica Gómez Fernández ${ }^{\star \star}$
}

\begin{abstract}
RESUMEN
Desde experiencias distintas de investigación e intervención, se revisarán metodologías utilizadas para el trabajo con la niñez. Los objetivos son dar a conocer herramientas e instrumentos y compartir retos metodológicos, tanto para la intervención como para la investigación con y desde los niños, niñas y adolescentes, incorporando metodologías participativas, inclusivas y lúdicas. También se releva la importancia que el juego tanto libre, como simbólico, tiene para la participación de estos actores en los estudios sociales y en la práctica diagnóstica e interventiva del Trabajo Social. Se apuesta aquí por una visión integral que permita reconocer e incluir a los niños y adolescentes, así como considerar sus grupos de relación socio-familiares. Además, se revisará el rol del /la facilitador o facilitadora en razón de cómo garantiza protagonismo, cómo se involucra en el juego y qué potenciales de cambio y resignificación puede derivar de dichas actividades.
\end{abstract}

Palabras clave: investigación, intervención, Trabajo Social, juegos, protagonismo.

\section{O jogo como metodologia de investigação e intervenção com crianças e adolescentes}

\section{RESUMO}

A partir de diferentes experiências de investigação e de intervenção, vão ser revistas metodologias utilizadas para o trabalho com infância. Os objetivos são apresentar ferramentas e instrumentos e compartilhar desafios metodológicos, tanto para a intervenção como também da investigação com e desde as crianças e adolescentes, incorporando metodologias participativas, inclusivas e lúdicas. Também se releva a importância que o jogo tanto livre, como simbólico, têm para a participação destes atores nos estudos sociais e na prática diagnóstica e interventiva do Trabalho Social. Aqui se aposta por uma visão integral que permita reconhecer e incluir as crianças e

* Artículo recibido el 2/07/2015. Artículo aceptado el 15/09/2015.

** Chilena. Trabajadora Social, académica del departamento de Gestión y Desarrollo Social de la Universidad Católica de la Santísima Concepción (UCSC).E mail: vegomez@ucsc.cl 
El juego como metodología de investigación e intervención con niñas, niños y adolescentes / Gómez

adolescentes, bem como considerar seus grupos de relação sócio familiares. Além disso, vão se revistar o papel do / a facilitador ou facilitadora em razão de como garante o protagonismo, como se envolve no jogo e as possibilidades de mudança e de re-significado que pode ser derivado de tais atividades.

Palavras-Chave: investigação, intervenção, Trabalho Social, jogos, protagonismo.

\section{The game as a methodology of research and intervention with children and adolescents}

\section{ABSTRACT}

From different experiences of research and intervention, this article will review methodologies for working with children. The objectives are to introduce tools and instruments and share methodological challenges for both the intervention and the research and from children and adolescents, incorporating participatory, inclusive and playful methodologies. It also shows the importance of the game, symbolic as well as free, for the participation of these actors in social studies and in diagnostic and interventional social work practice. It gives a comprehensive vision to recognize and include children and adolescents as well as considering their social and family relationship. In addition, the role of the facilitator is reviewed as how it ensures ownership, how is involved in the game and what potential changes and new meanings can be derived from such activities.

Keywords: Research, intervention, social work, games, role

\section{Introducción}

Existe un desconcertante desencuentro entre las formas de abordar a los niños y niñas en una investigación y en una intervención desde el Trabajo Social. Mientras en la primera, aún con poco asentamiento en la práctica, y especialmente relacionado con la entrevista (Navarro, 2000), es recurrente la alerta de la protección de los derechos con consentimientos informados y toda una procura de consentimientos adicionales; en la intervención con niños, niñas y adolescentes se ha estado asentando una práctica que los ha tomado como objetos sobre los que aplicar herramientas y acciones, en nombre de ellos y por su propio supuesto beneficio. Habitualmente, lo anterior no es más que el beneficio de estructuras de funcionamiento y de los adultos que planean y aplican Políticas Sociales, ya sean estas de servicios sociales, judiciales, 
educativos o de salud, por nombrar las que más relación directa pueden tener con los niños, niñas y adolescentes. Es una fórmula planteada desde intereses adultos donde los niños tienen siempre las de perder: "Interpretar las diferencias respecto a los estándares de racionalidad de los adultos siempre es desventaja para los niños" (Lowy, 1992).

El respeto real de los Derechos y el Interés Superior del Niño (ISN) (Naciones Unidas, Convención de los Derechos del Niño, 2013) son asignaturas pendientes en los procesos interventivos. La deuda pendiente es un planteamiento muy distinto de las formas de abordar las necesidades sociales y las problemáticas que afectan a nuestro mundo y modos de vida, un paso hacia la comprensión de una diversidad poco reconocida y aceptada como es que no todos los símbolos y significados se representan iguales para los niños, niñas y los adultos. La intervención participativa con niños y niñas, entonces, comprende también la intervención situada y contextualizada de acuerdo a las características de éstos, no sólo en su diseño, sino también en su abordaje práctico. Nos vendría de mucha ayuda poder visualizar o identificar si nuestros esfuerzos en el proceso de intervención en favor de la protección infantil y cómo se entiende ésta, generan riesgos sobre los cuales no hemos reparado lo suficiente; nuestras contradicciones entre el diseño y la ejecución, nuestra ideología de la infancia, la participación utilitarista, entre otros. Debemos, pues, comenzar a revertir nuestras prácticas profesionales en los ámbitos de la intervención social, e implicar progresivamente nuevas formas de entender, comunicar $\mathrm{y}$ transformar situaciones indeseables que comprometen a niños, niñas y adolescentes más allá de las circunstancias del orden social y político, el devenir histórico y los intereses de los grupos dominantes; pero ¿y en relación a la investigación? ¿Podemos construir formas de implicación de los niños y niñas con el cuidado necesario, pero sin que por ello, de nuevo, y ahora por otras razones, queden excluidos?

Una investigación implicativa, inclusiva, es ante todo una investigación hecha con sujetos (Ruano, 2000; Clifford, 2001; Ibáñez, 
1998), sean estos adultos o niños. Para ello, en ambos casos, los estudios se convierten en procesos de encuentro entre sujetos movidos y conmovidos por el objeto de estudio, es decir, por el tema de investigación. Los niños, niñas y adolescentes, desde sus particulares formas de comprender el mundo, construyen una relación con el tema de estudio; se lo reconozcamos los adultos, o no, también son actores de sus vivencias y relaciones, con posiciones y opiniones concretas sobre ellas.

Comprender, entonces, una investigación e intervención desde estos parámetros complejos de reflexión y participación de los niños y niñas con los que trabajamos en sus procesos de convivencia, requiere también nuevas formas de aproximación, con metodologías y con éticas atingentes. Para ello, debemos tener en cuenta particularidades psico-evolutivas, pero también las de género, socioculturales y de convivencia.

Para poder responder a estos desafíos se propone dar énfasis especial al juego como metodología de intervención e investigación; un medio natural de expresión de la niñez en complemento a otras técnicas, convencidas de que todo juego significa algo y que estos significados conectan a los seres humanos, de una forma $u$ otra. Jugar es una actividad propia de la niñez, es alegre y agradable, desarrolla habilidades para luego comunicarnos, aprender y crecer. El jugar conecta a los niños y niñas con otros en forma positiva, estimula el pensamiento y la exploración del mundo, regula emociones y alimenta el ego (Landreth, 2002). Además, el jugar permite practicar habilidades y roles necesarios para sobrevivir. El aprendizaje y el desarrollo son fomentados mejor a través del juego (Russ, 2004).

\section{Visualizar experiencias}

A continuación, se presentan algunas experiencias realizadas con herramientas lúdicas como instrumentos primordiales, aunque no los únicos, siempre sin perder el norte colaborativo que toda tarea debe integrar. Aunque son escasos los ejemplos de implicación de los niños y niñas en todo el proceso de investigación, qui- 
siera rescatar la experiencia investigativa con niños, niñas y adolescentes desarrollada por la Trabajadora Social María Belén Ortega Senet, de la UCSC, en Chile (Ortega, 2012); donde los niños, niñas y adolescentes son considerados como protagonistas de sus procesos, actores relevantes en la transformación de la realidad y potentes narradores de su historia y experiencias. El objetivo era conocer las percepciones de los niños y niñas en elementos claves en el tema de investigación: identidades, problemas de convivencia barrial, tomas de decisiones, organización en relación al género, el poder y la participación, incluyéndolos en la construcción del objeto de estudio, y en el proceso de investigación. La autora propone llevar a cabo actividades con niños entre los 10 y los 16 años, con juegos grupales como por ejemplo, "El espectáculo"; cuyo objetivo es observar la organización y procesos, formas de tomar decisiones, ofreciendo la posibilidad de realizar un proceso de investigación.

Otra actividad interesante es la referida al ejercicio individual "Este soy yo", cuyo objetivo es observar cómo se identifican los niños, niñas y adolescentes a sí mismos en varios niveles: cognitivo, identitario y de habilidades. Las posibilidades investigativas son múltiples, dado que se puede determinar el énfasis, y los resultados pueden servir tanto como datos como para poder determinar unidades de estudio y definir el tema de investigación.

Con el objetivo de conocer la percepción y valoración que los niños tienen de un tema determinado que acontece en su vida cotidiana, se realiza "El juego grupal"; aquí las posibilidades son inmensas y dependerán en cada caso de las particularidades del grupo, y se dispone de un nivel de organización de toma de decisiones y otro de visiones del problema tratado, de los personajes involucrados y de valores que se adscriben a cada propuesta de solución.

Entre las actividades con adolescentes entre los 13 y los 16 años en el proceso investigativo, se destaca la conformación de un "GIP" (Grupo de Investigación Participante) con el que diseñó e implementó varias tareas de investigación, como por ejemplo: La entre- 
vista entre los pares; un autoanálisis de los discursos y Las fotos en espacios cotidianos y significativos, en los que el niño, niña o adolescente puede retratar las cosas que para su vida son importantes.

Con los niños mayores y adolescentes se recomienda también video foros o grupos de discusión, los cuales constituyeron recursos importantes para lograr implicar a los jóvenes en el proceso y conseguir un acercamiento real a sus visiones, expectativas y valoraciones, pero la clave de lo valioso que pueden llegar a ser estos métodos e instrumentos de investigación, es cómo se lleva a cabo este encuentro entre adultos y niños y cuán aceptados y valorados se sienten ellos como participantes en el proceso al que se les invita.

Las propuestas para la intervención, nacen de las estrategias implementadas para la intervención familiar breve de prevención comunitaria y de educación inicial en programas de la Fundación "Hogar de Cristo" del Gran Concepción, para acompañar procesos de niños y niñas a contar del año 2010. Lo anterior surge a raíz de la toma de conciencia de las fortalezas que el juego libre y el juego simbólico tienen como construcción de la realidad.

A partir de la reflexión de los equipos de trabajo, se evidencia la existencia de espacios dentro de las instituciones orientados al ocio y la recreación de los niños y niñas, que no eran considerados ni aprovechados para la relación constructiva y para establecer un diálogo desde códigos comprendidos y perfectamente manejados por ellos. También pudimos entender que el juego va más allá del espacio de ocio, constituyéndose como el medio natural de expresión de los niños y niñas, mediante el cual manifiesta sus intereses, aspectos de su historia personal y se liberan sentimientos respecto a alguna situación vivenciada. No es novedad que el juego es una instancia que entrega valiosa información para el acompañamiento de los niños y niñas y es un aporte a la resignificación y reducción de vivencias dolorosas, aliviando la ansiedad y disminuyendo distintos temores.

Algunas experiencias de intervención con niños y niñas que se han utilizado en el trabajo con vulneraciones de derechos de baja 
y mediana complejidad en el contexto de programas de intervención breve, han contribuido a incorporar amigablemente la mirada de los niños y niñas en la construcción de su diagnóstico familiar desde metodologías más pertinentes. He aquí algunas de ellas.

Genograma animal: Tiene como objetivo incorporar la visión de las relaciones y rutinas familiares desde la perspectiva de los niños y niñas. Para esto, se le solicita al niño o niña que realice un dibujo de su familia, identificando a las personas, luego por cada una de ellas debe escoger un animal que la represente. Se le invita a compartir por qué escogió a determinado animal para la persona y si le parece, que invente una historia con los animales escogidos.

Entre las posibilidades de uso se reconoce la observación de las dinámicas familiares; interacciones y nivel de contacto entre los miembros de la familia, estilos de crianza, entre otros. Para el niño y niña, le ofrece a través del uso de la metáfora, expresar tensiones emocionales, temores, conflictos y agresiones.

Máscara de emociones: Una estrategia para trabajar principalmente con adolescentes cuyo objetivo principal es contribuir a fortalecer la expresión emocional de los y las jóvenes como factor de resiliencia. Se trabaja con diversos materiales atractivos para ellos y ellas, como cartulinas, lápices y todo tipo de desechos reutilizables que eligen y construyen una máscara, o varias, a través de las cuales se expresa alguna conducta o característica de personalidad propia. A cada una les pone su nombre y un apellido "La Verónica de azúcar" y comenta cómo es ese personaje. Posteriormente, se le invita y motiva a que refiera sus sentimientos negativos y positivos hacia esa máscara. Las posibilidades observadas de esta técnica son que los y las adolescentes puedan aprender a experimentar y expresar emociones de manera indirecta; el uso de máscaras otorga distancia y, por ende, son un medio menos amenazante y más seguro para abordar temas que no pueden ser planteados en forma directa y explícita.

El acuario familiar: Técnica que puede ser trabajada con un grupo familiar, incorporando la visión de las dinámicas familiares des- 
de la perspectiva de los niños y niñas. Permite dos tipos de análisis, primero, de la actitud frente a la realización de la tarea por parte de cada uno de los miembros de la familia y del nivel y tipo de comunicación verbal y no verbal que utiliza el grupo, y otro que observa principalmente los relatos asociados a las metáforas y símbolos utilizados. Si dentro de la familia existiera un agresor, debe evitarse la participación de éste en el contexto de juego familiar. En primer lugar, se les solicita a los integrantes de la familia presentes que con los materiales entregados y según su elección, realicen un acuario con especies marinas en el cual estén todos representados. Cada miembro debe escoger una especie que lo represente y consensuar su ubicación dentro del acuario en relación con otros. Luego deben explicar el porqué de cada elección y su posición en el acuario. Entre las posibilidades que se ofrecen con esta técnica se encuentran la observación de las dinámicas familiares respecto a la habilidad y deseo de organizarse frente a la tarea; nivel de contacto entre los miembros de la familia; nivel de disfrute de la familia; nivel de insight logrado, entre otras.

Una estrategia con grupos de pares: El retorno a la alegría - cartillas de juego; cuyo objetivo de implementación fue aportar en la disminución de las afectaciones emocionales de los problemas psicoafectivos que presentaban los niños y las niñas, en contexto post terremoto, Chile 2010. Estas técnicas de juego grupal constan de nueve unidades, y cada unidad presenta 4 talleres lúdicos recreativos, el último taller de cada unidad son juegos dinámicos activos para fortalecer el trabajo en equipo y conocer un poco el liderazgo que hay en cada niño y niña. A modo de ejemplo, el juego de confianza "submarinos amigos", en el cual se forman grupos de tres o cuatro niños y niñas en fila y tomados por los hombros. Se les indica que imaginen que son un submarino, con los ojos cerrados, menos la última persona que, al ser periscopio, ve todo el camino y guía al resto de la siguiente manera: Si da un apretón al hombro a la derecha deben girar a la derecha; un apretón a la izquierda se gira en este sentido; si toca la cabeza el submarino tendrá que pararse; el periscopio pasa el mensaje a quien está ade- 
lante. Esta persona habrá de transmitir el mensaje recibido a la persona siguiente y así sucesivamente, de esta forma el submarino avanzará intentando no chocar con otros submarinos ni objetos. Al cabo de un rato, quien está en cabeza se pone de periscopio. Así hasta que todos llegan a ser periscopio y cabeza en momentos diferentes. Una variante utilizada fue poner obstáculos para que los periscopios sean capaces de conducir a sus submarinos sin tropezar.

Las posibilidades de este simple juego son desarrollar el respeto y la aceptación de sí mismo y de los otros; desarrollar sus propias habilidades y por lo tanto, una mayor seguridad con relación a ellas.

\section{Valor del uso del juego simbólico como abordaje sistémico}

La revisión de las experiencias anteriores corrobora la idea de que la utilización del juego como principal herramienta en el trabajo con los niños y las niñas promueve, sin duda, un mayor bienestar emocional de una manera natural, menos invasiva y más contextualizada, ya que a través del juego exploran el mundo, desarrollan sus emociones y psiquis, como lo avalan diversos estudios en neurociencias y, además, someten a prueba la realidad y exploran conductas y roles. Permite la consideración de los niños y niñas en respeto a su dignidad humana como sujetos efectivos de derechos y como sujetos sociales.

La intervención lúdica no es cualquier cosa, es un abordaje estructurado basado en la teoría, que se construye sobre los procesos comunicativos y de aprendizaje normales de los niños (Carmichael, 2006; Landreth, 2002). Por otro lado, las investigaciones en terapia de juego, desde hace varios años, apoyan su efectividad en niños que experimentan una amplia variedad de problemas sociales, emocionales, del comportamiento y de aprendizaje, incluyendo niños cuyos problemas están relacionados al estrés causado por divorcio, muerte, mudanza a otra ciudad, hospitalización, enfermedades 
crónicas, experiencias tensionantes, abuso físico y sexual, violencia doméstica y desastres naturales (Reddy, Files-Hall \& Schaefer, 2005). A decir de Maturana: "el niño está donde está su emoción”.

Habitualmente los niños y niñas juegan libremente en su contexto familiar y /o social y entendemos que ello proyecta el mundo interior de la persona y desarrolla la psiquis de todo ser humano. Sin embargo, también se puede incorporar en la intervención con niños y niñas el juego simbólico, basado en la interpretación de Piaget que señala que la aparición, desarrollo y evolución de la función simbólica representa algo por medio de un significante diferenciado, es decir, el niño o niña puede distanciarse de la realidad para crear una situación ficticia sin mayor dificultad. Desde el enfoque sistémico, cualquier problema expresado por los niños y niñas como síntoma, es de la familia. En esta mirada, la incorporación estructurada e intencionada de distintos miembros del sistema familiar a las actividades de juego permite al facilitador (a) observar, evaluar y comprender el funcionamiento del sistema, pero también genera conciencia en ellos mismos, facilitando la promoción de cambios en las interacciones familiares a través del juego. En la terapia familiar sistémica, el juego opera como elemento reparador en sí mismo, implicando a la familia en una instancia de conexión positiva, en la cual también se ponen en práctica habilidades para la vida.

En situaciones de vulneraciones de derecho, genera espacios para que el niño o niña experimente junto a sus cuidadores momentos felices o de menor tensión y pueda comunicar en situación de menor estrés, lo que le incomoda o dificulta. Aquí cobra vital importancia la observación del lenguaje metafórico, la comunicación simbólica y experiencias placenteras (Reyes, 2011).

\section{El rol del facilitador}

El juego es una vía de expresión humana y natural del niño y la niña, y restringirlo a una expresión de tipo verbal en situaciones principalmente diseñadas para y por los adultos limita la inter- 
vención y la investigación, ya que establece automáticamente una barrera a la comunicación entre los niños y niñas y nosotros, los facilitadores. Esto implica transmitirle a un niño: 'Tú debes ponerte a mi nivel y comunicarte a través de palabras y en las circunstancias que yo establezco', desconociendo que el juego debe ser fundamentalmente controlado por el jugador.

El papel del educador (a) ante cualquier actividad del niño "debe ser de permitir, favorecer, observar, registrar, documentar y posteriormente reflexionar sobre ellas para reconocerlas, sacar conclusiones y darles un sentido" (Ruiz de Velasco, 2006). No obstante, no se debe olvidar que todos los observadores recogemos idéntica información, lo anterior, mediado por nuestras propias experiencias e interpretaciones.

Es responsabilidad del investigador, o del terapeuta, preparar el contexto del juego y la propia disposición; ponerse en el nivel de comunicación de los niños y niñas a través de un medio que les resulte cómodo, dar seguridad física y afectiva y expresar la empatía tónica que es capaz de esperar y ajustar. No se trata de negar la influencia del adulto que interviene y su poder encubierto, es más bien tener autoconciencia respecto a la propia situación emocional. ¿Por qué el niño se debe acomodar al adulto? "Se supone que es el adulto el que logra comunicarse en todos los niveles y el que tiene una comprensión evolutiva de los niños" (Landreth, 2002). Lo anterior, supone no restringir el juego a lo que es útil o no para el adulto y a un afán de democratización de los espacios de actuación.

El facilitador debe ser capaz de involucrarse en el juego y crear una relación de seguridad que favorezca la exploración de aquellos asuntos que afectan su vida, mostrar siempre una actitud de comprensión para lograr involucrarse en el juego y crear una relación de seguridad que favorezca la exploración. También debe procurar saber ser uno de ellos, cuando ha lugar, con la aceptación absoluta del niño y sus circunstancias, el respeto a sus derechos, teniendo en cuenta su interés superior. Todo ello facilita el proceso del vínculo y construye una relación positiva durante las sesiones. 
Es conveniente contar con una buena batería de juegos y dinámicas que pueden ser modificadas según los intereses, objetivos y características de los niños y grupos de niños. Para ello, es importante una buena dosis de creatividad y la flexibilidad en los usos de técnicas de juegos que pueden complementarse con otras técnicas.

\section{Conclusiones}

La presentación de estas experiencias es relevante porque han sido provechosas para el trabajo de intervención con niños, niñas y adolescentes. Cada una de las propuestas acerca de un modo diferente a ellos y ellas, a sus hábitos y cotidianidades, a sus formas de hacer, sentir y pensar, a cómo reflexionan su vida, sus puntos de vista y percepciones subjetivas, todo junto y enredado ya que en el juego "existe un pensamiento circular puesto de manifiesto: sentir, pensar, actuar, es un hecho circular, no se siente o luego se piensa y luego se actúa. Se actúa, se piensa y se siente a la vez" (Maturana y Varela, 1998).

La riqueza de los resultados nos posiciona en lugares privilegiados para comprender de manera más profunda a los niños y niñas y sus sistemas de relación y los contextos, el proceder y las dinámicas en la intervención desde el Trabajo Social. Nos permite detenernos más en sus competencias y capacidades, en sus fortalezas y resiliencia y de ese modo, ser más coherentes con el sentido de justicia. Pero además, estas maneras gratas de acercarse a los sujetos de investigación y/o intervención, se convierten en algo más que sus propósitos, crea lazos y curiosidades mutuas, conformando al juego, finalmente, como un marco de encuentro e intercambio para todos, ellos, ellas y nosotros.

\section{Bibliografía}

Clifford, J. (2001 (1º ed. 1995)). Dilemas de la cultura. Antropología, literatura y arte en la perspectiva postmoderna. Barcelona: Editorial Gedisa.

Garvey, C. (1985). El juego infantil. Madrid: Ed. Morata S.A. 
Ibáñez, J. (1998). Nuevos Avances en la Investigación Social, I. Ed. Proyecto a. Barcelona: Cuadernos Biblioteca Universitaria.

Landreth, G. (2002). Play therapy: The art of the relationship. NewYork: Brunner-Routledge.

Liebel, M. (2013). Niñez y justicia social, repensando sus derechos. Pehuén editor. Santiago de Chile.

Lowy, Catherine (1992). Autonomy and the appropriate projects of children: a comment on freeman, en Ph.alston, S.Parker \& J.A.Seymour (eds.) Children, Rights, and the Law. Oxford University Press, pp. 72-75.

Maturana, H. y Verden- Zöller Gerda (2007). Amor y Juego: Fundamento Olvidados de lo Humano, desde el patriarcado a la democracia. Santiago: Editorial C.J. Sáez.

Naciones Unidas, Convención sobre los Derechos del Niño (2013). Observación general $N^{\circ} 14$ sobre el derecho del niño a que su interés superior sea una consideración primordial, 201. Recuperado de http://www2.ohchr.org/English/bodies/crc/ docs/GC/CRC.C.GC.14_sp.pdf

Navarro Sada, A. (2000). Estándares éticos para la investigación con niños según la Society for Research in Child Development. Recuperado de http://www.uam.es/personal_pdi/stmaria/alenava/Docencia/DOCTORADO/PRIMERA_PARTE_DOCS/ESTANDARES_ETICOS.pdf

Ortega, M.B. (2012). Jóvenes y poder local en el proceso de cambio boliviano (2008-2010). Reflexiones sobre la agencia de jóvenes participantes del taller de formación política del proyecto de poder local en El Alto, Bolivia. Tarragona, Cataluña. Universitat Rovira i virgili. Tesis doctoral. Recuperado de http://biblioteca.universia.net/utml_bura/ficha/params/ litle/jovenes-poder-local-proceso-cambio-boliviano2088-2010-reflexiones-agencia/id/58741820.htm.

Ruano, L. (2000). De la construcción de los otros por nosotros a la construcción del nos-otros. Revista de Educación Nueva Época, (12):1-8, Guadalajara, Jalisco CP.

Ruiz, A y Abad, J (2011). El juego simbólico. Barcelona: Editorial GRAÓ, de IRIF, S.L.

Russ, S. W. (2004). In child development and psychotherapy. Mahwah, NJ: Lawrence Erlbaum Associates Publishers. Recuperado de http://psycnet.apa.org/psycinfo/2003-88219-000

Shaefer, C. (2012). Fundamentos de terapia de juego. México: Ed. Manual moderno. Recuperado de http://libros.elmundo.es. 
